\title{
Identificación y análisis del aprendizaje del judo mediante la metodología observacional
}

\author{
Identification and Analysis of Learning Judo by Observation Method
}

\author{
ALFONSO GUTIÉRREZ SANTIAGO \\ IVÁN PRIETO LAGE \\ Facultad de Ciencias de la Educación y del Deporte (Pontevedra) \\ Universidad de Vigo
}

\section{OLEGUER CAMERINO FOGUET}

Laboratorio de Observación de la Motricidad

INEFC-Universidad de Lleida

\section{MARÍA TERESA ANGUERA ARGILAGA}

Facultad de Psicología

Departamento de Metodología de las Ciencias del Comportamiento

Correspondencia con autor

Universidad de Barcelona

Oleguer Camerino Foguet

ocamerino@inefc.es

\begin{abstract}
Resumen
Este artículo estudia los factores que intervienen en el aprendizaje de los deportes de combate a partir de un estudio observacional de los errores técnicos y sus relaciones. Nos centramos en la ejecución del modelo técnico de una de las proyecciones de judo ( $O$ Goshi) por parte de una muestra de 55 estudiantes de la Licenciatura en Ciencias de la Actividad Física y del Deporte de la Universidad de Vigo a lo largo de cinco cursos académicos. Para ello, se ha realizado un análisis observacional mediante un instrumento de observación (SOBJUDO-OG) y un instrumento de registro Match Vision Studio Premium v.1.0. software. Los resultados, determinados mediante estadística descriptiva y análisis secuencial de T-Patterns obtenido mediante el programa Theme 5.0 software, corroboran que la falta de flexión de piernas provoca una ausencia de carga del cuerpo del adversario -Uke- sobre la propia cadera del ejecutante -Tori-, y que la falta de desequilibrio inicial con ambos brazos o la separación de la parte posterior de la cresta iliaca izquierda de Tori sobre la parte anterior del hemicuerpo izquierdo de Uke conduce también a la ausencia de dicha carga.
\end{abstract}

Palabras claves: judo, $O$ Goshi, observación deportes de combate, análisis secuencial con T-patterns

\section{Abstract \\ Identification and Analysis of Learning Judo by Observation Method}

This article examines the factors involved in learning combat sports based on an observational study of technical errors and their relationships. We focus on the implementation of the technical model of one of the throws of judo (O Goshi) using a sample of 55 students on the Bachelor of Science in Sport and Physical Activity at the University of Vigo over five academic years. To that end we carried out observational analysis using an observation instrument (SOBJUDO-OG) and a recording instrument (Match Vision Studio Premium v.1.0. software). The results, reached using descriptive statistics and sequential analysis with T-Patterns obtained using the Theme 5.0 software program, confirm that the lack of bend in the legs leads to an absence of load of the opponent's body - Uke - on the hip of the judoka carrying out the throw - Tori - and that lack of initial disequilibrium with both arms, or separation of the rear of the left iliac crest of the Tori on the front of the left half of the Uke's body also leads to the absence of this load.

Keywords: judo, O Goshi, combat sports observation, sequential analysis with T-Patterns 


\section{Introducción}

En el ámbito de la investigación deportiva se manifiesta una necesidad creciente de obtener de forma rigurosa datos que nos aporten evidencias empíricas sobre la existencia de regularidades no detectadas desde la inferencia visual. Es uno de los retos actuales la búsqueda de nuevas metodologías de análisis y la construcción de instrumentos de observación ad hoc, combinados con registros de codificación informatizada, considerados todos ellos desde un planteamiento propio, sobre contextos naturales del deporte (Castañer, Torrents, Dinušová, \& Anguera, 2009; Jonsson et al., 2006).

Existe la necesidad de explicar, predecir o incluso controlar los factores que condicionan el éxito en el proceso de enseñanza y aprendizaje de la técnica deportiva (Godoy, 1994; Oña, Martínez, Moreno, \& Ruiz, 1999; Ruiz \& Sánchez, 1997; Schmidt \& Lee, 2005). El estudio del conocimiento de la ejecución de la técnica de los deportes de combate, y en particular el de los errores técnicos cometidos en el judo es una herramienta útil y novedosa (Gutiérrez \& Prieto, 2006, 2007a, 2007b). Todos estos estudios corroboran las conclusiones de Gentile (1972) que apunta que la corrección de un movimiento o gesto técnico desde la perspectiva del error, resulta más útil si existe una información sobre la naturaleza de los errores cometidos que simplemente señalar el resultado de la ejecución.

La utilización de la técnica $O$ Goshi en el ámbito de la iniciación al judo ha sido, desde su creación -en 1882- hasta la actualidad, una constante en la mayoría de las propuestas para su enseñanza (Castarlenas \& Calmet, 1999; García, Sterkowicz, \& Carratalá, 2003), las cuales han establecido también una organización específica del aprendizaje del judo en la que $O$ Goshi está siempre presente dentro de los primeros pasos de la iniciación.

El análisis del error técnico de $O$ Goshi a partir de la metodología observacional (Anguera, Blanco, \& Losada, 2001) nos ha permitido, alcanzar dos objetivos fundamentales: obtener la frecuencia de los errores durante la ejecución de la técnica $O$ Goshi, y comprobar la presencia de relaciones importantes entre estos y constatar la existencia de patrones secuenciales que determinen la relación entre los errores.

El propósito final de la presente investigación es analizar los errores más frecuentes que se producen en la ejecución de la técnica de proyección $O$ Goshi en alumnos universitarios, evidenciando las relaciones existentes entre ellos, y de este modo, establecer una herramienta que sirva a los distintos profesionales del judo como soporte técnico en el proceso de enseñanza-aprendizaje.

\section{Método}

Se ha utilizado la metodología observacional (Anguera, 1999; Anguera et al., 2001) que goza del necesario rigor y flexibilidad para estudiar los episodios que se presentan de forma natural en el proceso de enseñanza aprendizaje de la técnica de judo. Basándonos en Heinemann (2003), podemos manifestar que el tipo de observación llevada a cabo ha sido estandarizada, abierta y no participante.

\section{Diseño}

Este estudio forma parte de un proyecto de investigación más amplio relativo a la detección de los errores más comunes en diversos gestos técnicos básicos en la iniciación al judo. El diseño observacional (Anguera et al., 2001) es nomotético (varios sujetos que ejecutan un misma técnica -O Goshi-), seguimiento (un gesto técnico desarrollado durante cinco cursos académicos), y multidimensional (las dimensiones se corresponden con los criterios del instrumento de observación). De este diseño $\mathrm{N} / \mathrm{S} / \mathrm{M}$ (nomotético/seguimiento/multidimensional) se derivan una serie de decisiones sobre los participantes, los instrumentos de observación-registro, y el procedimiento de análisis.

\section{Participantes}

El presente estudio se ha realizado en la Facultad de Ciencias de la Educación y del Deporte de la
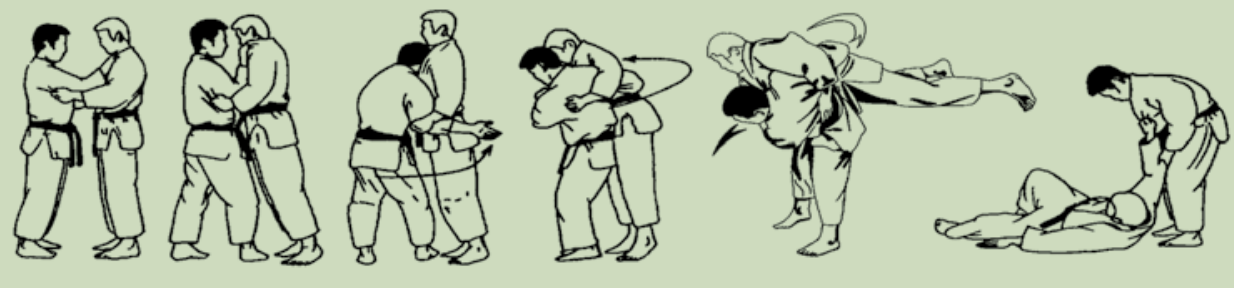

4 Figura 1 Técnica O Goshi (Inogai \& Habersetzer, 2002) 
Universidad de Vigo, utilizando para ello dos tatamis diferentes de $10 \times 10$ metros, ubicados en el Centro Gallego de Tecnificación Deportiva y en la propia facultad.

La muestra ha sido segmentada en base a unos criterios de selección. Para esta investigación hemos seleccionado 55 sujetos aleatoriamente, 25 hombres y $30 \mathrm{mu}-$ jeres, alumnos de la Licenciatura en Ciencias de la Actividad Física y del Deporte, neófitos en esta disciplina y pertenecientes a los cursos académicos 2003/2004, 2004/2005, 2005/2006, 2006/2007 y 2007/2008. Se hicieron en total 55 registros distribuidos equitativamente entre los cursos.

\section{Procedimiento}

La ejecución de la técnica objeto de estudio - $O$ Goshi- fue filmada tras finalizar un periodo de formación de aproximadamente cuatro meses. La recopilación de datos se ha realizado mediante una grabación con dos cámaras de vídeo digital (Samsung VP-D70 y Samsung VP-D301 -cursos 2003/04 a 2005/2006- y JVC GZ-MG21E -cursos 2006/07 a 2007/08-), utilizando en ambos casos un plano entero con un enfoque normal (Lomas, 1996). Estas se han dispuesto sobre dos trípodes, a una altura de 1,50 metros, situadas en ángulo una frente a la otra y aproximadamente a 2 metros del tatami. A continuación, se realizó una edición de las distintas proyecciones filmadas mediante el programa informático de edición de vídeo Pinnacle Studio (versiones $8,9,11$ y 12 ).

\section{Instrumento de observación}

El instrumento de observación que hemos construido para este estudio es el SOBJUDO-OG que sigue el mismo rigor metodológico de otros estudios (Fernández, Camerino, Anguera, \& Jonsson, 2009; Gutiérrez, Prieto, \& Cancela, 2009) ya que contempla en sus criterios todos los errores técnicos en la ejecución de $O$ Gosh $i$ y su elaboración se ha realizado a partir de la descripción del modelo técnico de proyección $O$ Goshi (adaptado de Taira, Herguedas, \& Román, 1992) que describimos a continuación:

Los judokas se encuentran uno frente al otro. El ejecutante -Tori- realiza un desequilibrio hacia delante con ambas manos; concretamente, levanta y tira con su mano izquierda, que se encuentra a la altura del codo, en la parte exterior de la manga de su adversario, al mismo tiempo que atrae hacia sí mismo el cuerpo de Uke con su mano derecha, que se sitúa en la línea imaginaria que une su hombro con la solapa del judogi de Uke, con lo que provoca el desequilibrio frontal de su compañero.

A continuación, Tori, a la vez que baja el centro de gravedad de su cuerpo, lleva su pie derecho a la parte interior -plano frontal- del pie derecho de Uke y a la misma altura -plano sagital-, aunque un poco adelantado $-20 \mathrm{~cm}$ a lo sumo-. Simultáneamente, introduce su brazo derecho por debajo de la axila izquierda de Uke, para acabar rodeando totalmente su cadera a la altura del cinturón o región lumbar.

Desde esta situación y mediante un giro en el eje longitudinal coloca su pie izquierdo en la parte interior -plano frontal- del pie izquierdo de Uke y a la misma altura -plano sagital- que su otro pie, quedando de espaldas al cuerpo de Uke, y manteniendo un estrecho contacto entre la parte posterior de la cadera de Tori y la anterior de Uke. Además, sin solución de continuidad, el ejecutante baja aún más su centro de gravedad por medio de una flexión de sus piernas, quedando la parte posterior de la cadera de Tori al nivel de los muslos de Uke. De este modo, Tori carga sobre su cadera el cuerpo de su compañero - gracias a la acción del brazo derecho-, a la vez que efectúa una ligera torsión de su tronco hacia el lado izquierdo y una elevación de su cadera, realizando un vigoroso movimiento de extensión de sus rodillas, lo que sumado a una gran flexión de su tronco favorece la proyección de Uke hacia delante en el plano sagital.

Finalmente, Tori, manteniéndose estable sobre sus pies, recupera la posición erguida de su tronco y tira con su mano izquierda hacia arriba del judogi del compañero para controlar la caída de este. La mano derecha ayudará en el control final de la proyección.

El SOBJUDO-OG (ver tabla 1) se ajusta al diseño observacional planteado, siendo de carácter multidimensional y constando de los siguientes criterios:

- Criterios fijos (CF): curso y sexo.

- Criterios cambiantes: agarre, desequilibrio, posición del pie derecho, posición del pie izquierdo, posición del brazo derecho, posición de la cadera, acción de piernas (A. P.), acción de carga (A. C.), fase de proyección (F. P.), maniobra de reequilibrio y globalidad (G.).

Cada una de los criterios da lugar a un catálogo de categorías que cumplen las condiciones de exhaustividad y mutua exclusividad (E/ME) y que da lugar a la 


\begin{tabular}{|c|c|c|}
\hline & Código & Descripción \\
\hline \multirow{3}{*}{ 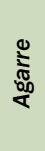 } & AGB & Tori agarra con su mano izquierda el judogi de Uke a la altura de la porción media del bíceps braquial. \\
\hline & AGM & Tori agarra con su mano izquierda la muñeca de Uke en la ejecución de la técnica. \\
\hline & AGBR & Tori agarra con su mano izquierda el judogi de Uke a la altura de la porción media del antebrazo. \\
\hline \multirow{4}{*}{ 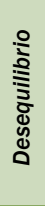 } & $\mathrm{DIZ}$ & La mano izquierda de Tori permanece en posición de agarre, sin efectuar ninguna acción de desequilibrio. \\
\hline & DIDE & La mano derecha de Tori permanece en posición de agarre, sin efectuar ninguna acción de desequilibrio. \\
\hline & DIZDE & Tori no desequilibra el cuerpo del adversario con ninguno de sus brazos. \\
\hline & DINC & La ejecución del desequilibrio y el posterior Tai Sabaki se efectúa con solución de continuidad. \\
\hline \multirow{3}{*}{$\begin{array}{l}\frac{2}{0} \\
\frac{\Phi}{0} \\
\frac{0}{0}\end{array}$} & PDDE & $\begin{array}{l}\text { Tori, tras el semigiro en el eje longitudinal -Tai Sabaki- sitúa su pie derecho inmediatamente delante -plano } \\
\text { sagital- del pie derecho de Uke, aunque a menos de } 20 \mathrm{~cm} \text {. }\end{array}$ \\
\hline & PDEX & $\begin{array}{l}\text { Tras el Tai Sabaki, Tori sitúa su pie derecho en la parte exterior -plano frontal- de la posición que ocupa el } \\
\text { mismo pie de Uke. }\end{array}$ \\
\hline & PDID & $\begin{array}{l}\text { Después del movimiento de Tai Sabaki, Tori ubica su pie derecho en el interior-plano frontal- de la posición que } \\
\text { ocupa el pie derecho de Uke, aunque adelantado - plano sagital- a más de } 20 \mathrm{~cm} \text { del pie de este. }\end{array}$ \\
\hline \multirow{7}{*}{$\frac{\substack{0 \\
\frac{0}{2}}}{\stackrel{\frac{0}{2}}{\frac{0}{2}}}$} & PINI & $\begin{array}{l}\text { El pie izquierdo de Tori permanece en la posición inicial, girando sobre sí mismo sin que se produzca desplaza- } \\
\text { miento alguno durante la ejecución de la técnica. }\end{array}$ \\
\hline & PID & $\begin{array}{l}\text { Tori, tras el Tai Sabaki, sitúa su pie izquierdo delante del pie izquierdo de Uke -plano sagital-, aunque a menos } \\
\text { de } 20 \mathrm{~cm} \text {. }\end{array}$ \\
\hline & PIDM & Tori sitúa su pie izquierdo delante del pie izquierdo de Uke -plano sagital-, aunque a más de $20 \mathrm{~cm}$. \\
\hline & PIEX & El ejecutante sitúa el pie izquierdo en el exterior -plano frontal- de la posición que ocupa el mismo pie de Uke. \\
\hline & PIID & $\begin{array}{l}\text { Después del Tai Sabaki, Tori coloca su pie izquierdo en el interior -plano frontal- de la posición que ocupa el pie } \\
\text { izquierdo de Uke, aunque adelantado -plano sagital- a más de } 20 \mathrm{~cm} \text { del pie de este. }\end{array}$ \\
\hline & PIDEX & $\begin{array}{l}\text { Tori sitúa su pie izquierdo inmediatamente delante -plano sagital- del pie izquierdo de Uke, aunque a menos } \\
\text { de } 20 \mathrm{~cm} \text { y exterior - plano frontal- de la posición que ocupa el mismo pie de Uke tras el semigiro en el eje } \\
\text { longitudinal. }\end{array}$ \\
\hline & PIDME & $\begin{array}{l}\text { El ejecutante sitúa su pie izquierdo inmediatamente delante -plano sagital- del pie izquierdo de Uke, aunque } \\
\text { a más de } 20 \mathrm{~cm} \text {. y exterior -plano frontal- de la posición que ocupa el mismo pie de Uke tras el Tai Sabaki. }\end{array}$ \\
\hline \multirow{4}{*}{ 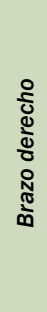 } & BCINT & $\begin{array}{l}\text { Tori, encontrándose de espaldas a Uke, y pasando su mano derecha por debajo de la axila izquierda de este, } \\
\text { coloca su mano derecha a la altura del cinturón de su adversario, pero en la mitad del cuerpo. }\end{array}$ \\
\hline & BESPA & $\begin{array}{l}\text { Tori pasa su mano derecha por debajo de la axila izquierda del cuerpo de Uke, abrazando la región dorsal de la } \\
\text { espalda de su adversario para proyectarle. }\end{array}$ \\
\hline & BCIPE & La mano derecha de Tori agarra el cinturón de Uke realizando una acción de “pesca” -Tsuri-. \\
\hline & BJUPE & $\begin{array}{l}\text { Tori pasa su mano derecha por debajo de la axila izquierda del cuerpo de Uke y agarra el judogi de su adversario } \\
\text { a la altura de la cintura, tirando de este mediante una acción de "pesca" -Tsuri-. }\end{array}$ \\
\hline \multirow{4}{*}{$\begin{array}{l}\frac{\pi}{0} \\
\frac{0}{0} \\
\frac{\pi}{0} \\
\frac{0}{0} \\
\frac{0}{0} \\
0\end{array}$} & CISE & $\begin{array}{l}\text { Existe una separación -plano sagital- entre la parte posterior de la cresta iliaca izquierda del cuerpo de Tori y la } \\
\text { parte anterior del hemicuerpo izquierdo de Uke durante la segunda fase de la proyección. }\end{array}$ \\
\hline & CIFU & $\begin{array}{l}\text { La parte izquierda de la cadera de Tori se encuentra fuera -plano frontal- del espacio comprendido entre las } \\
\text { dos crestas iliacas de Uke. }\end{array}$ \\
\hline & CDME & $\begin{array}{l}\text { La cresta iliaca derecha de Tori se encuentra en la parte media de la cadera de Uke, pero su cresta iliaca } \\
\text { izquierda, aunque se sitúa dentro de la línea de los pies de Uke -plano frontal- se encuentra separada -plano } \\
\text { sagital- del hemicuerpo izquierdo de Uke. }\end{array}$ \\
\hline & CDFU & $\begin{array}{l}\text { La parte derecha de la cadera de Tori se encuentra fuera -plano frontal- del espacio comprendido entre la ca- } \\
\text { dera de Uke y por detrás -plano sagital- del eje transversal de esta, y la cresta iliaca izquierda de Tori se ubica } \\
\text { enfrente de la parte media de la cadera de Uke y separada - plano sagital- del hemicuerpo izquierdo. }\end{array}$ \\
\hline
\end{tabular}

$\Delta$

Tabla 1

Sistema de categorías SOBJUDO-OG 


\begin{tabular}{|c|c|c|}
\hline & Código & Descripción \\
\hline \multirow{3}{*}{$\dot{a}$} & APEX & Las piernas de Tori permanecen en extensión durante toda la proyección. \\
\hline & APFNE & Tori, tras efectuar una flexión de piernas en el Tsukuri no realiza la extensión de las mismas. \\
\hline & APIF & Tori realiza una flexión insuficiente de piernas -su cadera no se sitúa a la altura de los muslos de Uke-. \\
\hline$\stackrel{0}{ن}$ & NCA & Tori, durante la ejecución de la acción, no carga en ningún momento el cuerpo de Uke sobre su cadera. \\
\hline \multirow{4}{*}{ 造 } & TNSI & Tori no simultanea la flexo-extensión de piernas y la tracción de brazos. \\
\hline & TAC & Tori acompaña la caída del cuerpo de Uke hasta el suelo con su brazo derecho. \\
\hline & TCCA & Durante la fase del Kake, Tori no controla la caída del cuerpo de su adversario. \\
\hline & TMFL & Tori realiza una flexión de tronco adecuada pero no recupera la verticalidad al finalizar la proyección. \\
\hline \multirow{5}{*}{ 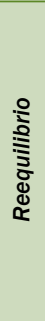 } & DA & Tori se desequilibra hacia atrás tras proyectar a su rival, corrigiendo su posición con la ayuda de su pie derecho. \\
\hline & DD & $\begin{array}{l}\text { Tori se desequilibra hacia delante tras proyectar a su rival, corrigiendo su posición con la ayuda de su pie } \\
\text { derecho. }\end{array}$ \\
\hline & AMD & $\begin{array}{l}\text { Tori se desequilibra hacia delante al realizar la técnica, corrigiendo su posición con la ayuda de su mano de- } \\
\text { recha. }\end{array}$ \\
\hline & IA & Después de ejecutar la proyección, Tori, apoya la pierna izquierda hacia atrás para reequilibrarse. \\
\hline & ID & Después de ejecutar la proyección, Tori, apoya la pierna izquierda hacia delante para reequilibrarse. \\
\hline ن & LENT & La ejecución de la proyección es lenta y sin continuidad. \\
\hline
\end{tabular}

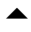

Tabla 1 (Continuación)

Sistema de categorías SOBJUDO-OG

construcción del siguiente sistema de categorías (ver tabla 1), que ejemplificamos con alguna imagen (fig. 2).

\section{Instrumento de registro}

Efectuamos un registro continuo a lo largo de la observación de todas las sesiones grabadas, y para ello hemos utilizado el programa informático Match Vision Studio Premium v.1.0. software (Castellano, Perea,
Alday, \& Hernández Mendo, 2008) (fig. 3). Este es un programa interactivo multimedia que permite visionar y registrar, en formato Avi, Mpg, en la misma pantalla del ordenador la grabación digitalizada de los videos.

Dicho programa es altamente flexible, permitiéndonos introducir las categorías de cada criterio cambiante del instrumento de observación SOBJUDO-OG ( $t a-$ bla 1), para así registrar su sucesión y cambios en la propia pantalla.

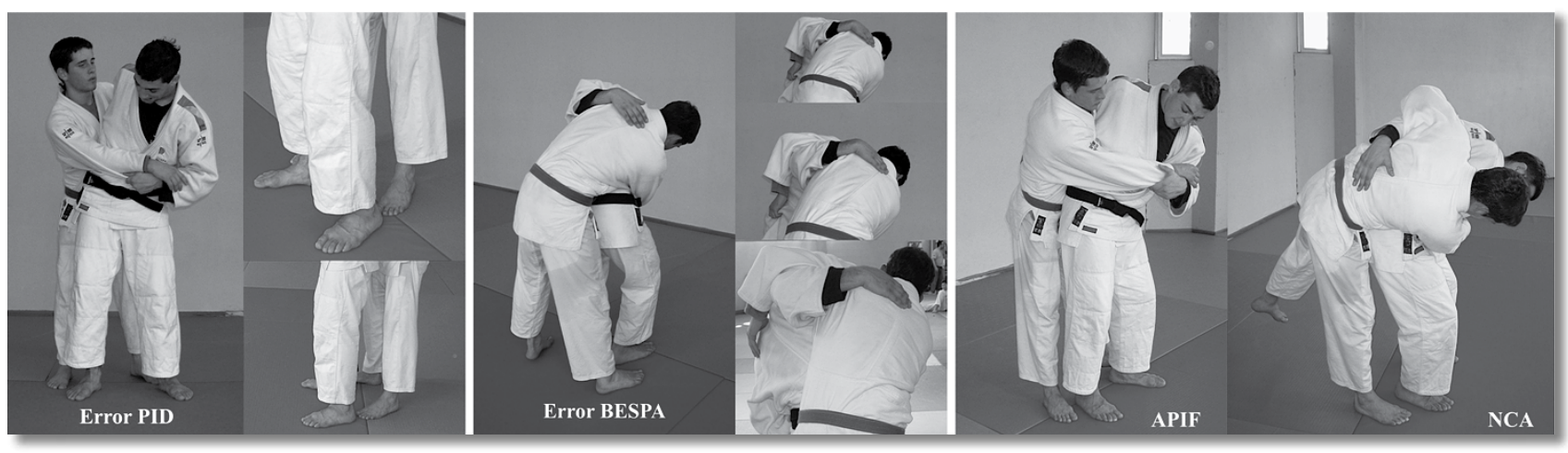

$\Delta$

Figura 2

Ejemplos de algunas categorías 


\section{Procedimiento}

La calidad de los datos (Blanco-Villaseñor \& Anguera, 2000) se han obtenido mediante la confección de un manual de la observación y el entrenamiento de dos observadores que han superado la fiabilidad interobservador con el coeficiente kappa -obtenido mediante el programa SDIS-GSEQ versión 4.2 (Bakeman \& Quera, 1992, 2001)- de un valor del 0,96 .

Después del registro de todas las acciones técnicas, obtenemos un archivo Excel (fig. 4) de las sucesivas configuraciones formadas por las líneas de códigos que han cambiado y su temporalidad y duración expresado en frames ( 25 frames equivalen a 1 segundo).

Estos archivos .xls nos han permitido disponer de las frecuencias de todas las ocurrencias de códigos registrados y han sido transformados sucesivamente para permitir diferentes análisis.

\section{Resultados}

\section{Análisis estadístico descriptivo}

El análisis descriptivo de frecuencias y porcentajes de ocurrencia de los errores técnicos (ver tabla 2) muestra una primera distribución de frecuencia y el porcentaje de los errores en el grupo de estudio $(n=55)$.

Del total de los 38 errores consensuados entre los observadores, casi la mitad de ellos son cometidos con una frecuencia muy baja -entre 1 y 5 sujetos únicamente-, por lo que, a priori, indicaremos que existen unos

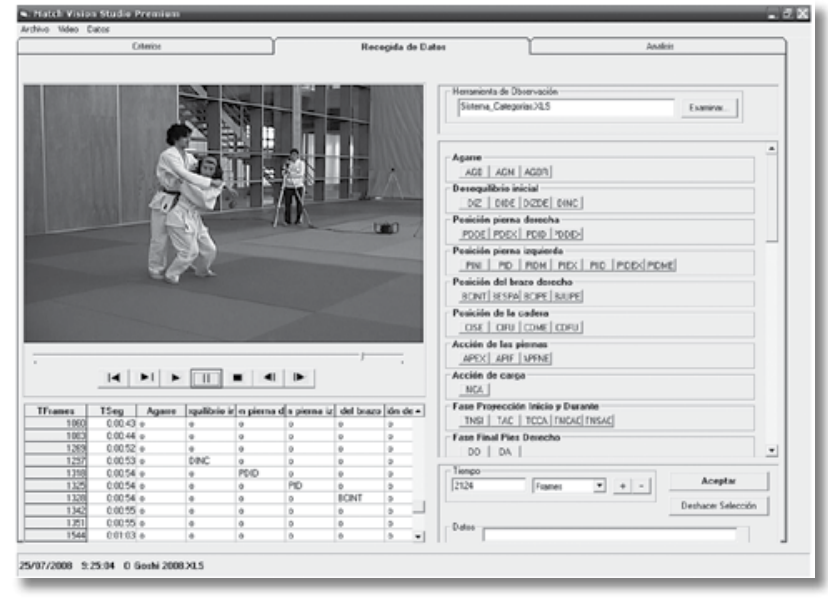

\section{Figura 3}

Instrumento de registro Match Vision Studio Premium v. 1.0 (Castellano et al., 2008)

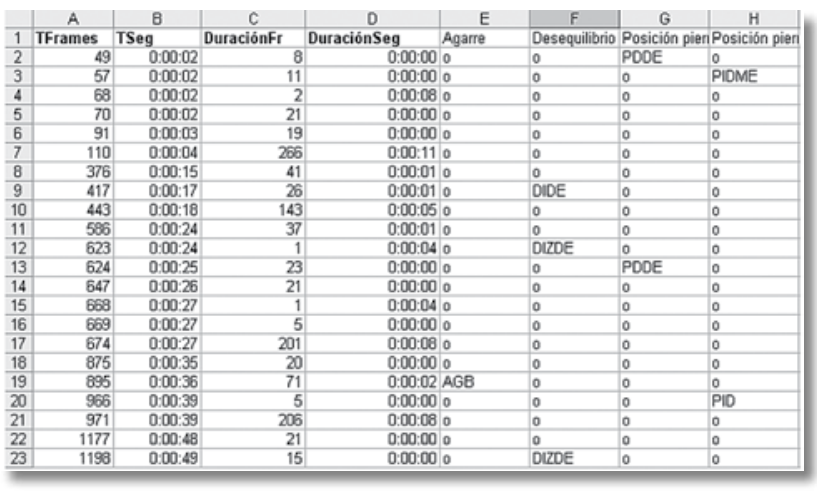

\section{Figura 4}

Registro en archivo .xls de los datos obtenidos mediante Match Vision Studio Premium v.1.0. (Castellano et al., 2008)

\begin{tabular}{|c|c|c|c|c|c|c|c|c|}
\hline Error & Frec. & Porcentaje & Error & Frec. & Porcentaje & Error & Frec. & Porcentaje \\
\hline \multicolumn{3}{|c|}{ AGARRE } & PIEX & 1 & $1,9 \%$ & APFNE & 4 & $7,4 \%$ \\
\hline AGB & 9 & $16,7 \%$ & PIID & 2 & $3,7 \%$ & \multicolumn{3}{|c|}{ ACCIÓN DE CARGA } \\
\hline AGM & 1 & $1,9 \%$ & PIDEX & 1 & $1,9 \%$ & NCA & 20 & $37 \%$ \\
\hline AGBR & 5 & $9,3 \%$ & PIDME & 6 & $11,1 \%$ & \multicolumn{3}{|c|}{ FASE DE PROYECCIÓN } \\
\hline \multicolumn{3}{|c|}{ DESEQUILIBRIO } & \multicolumn{3}{|c|}{ POSICIÓN BRAZO DERECHO } & TNSI & 2 & $3,7 \%$ \\
\hline DIZ & 2 & $3,7 \%$ & BCINT & 7 & $13 \%$ & TAC & 13 & $24,1 \%$ \\
\hline DIDE & 9 & $16,7 \%$ & BESPA & 19 & $31,2 \%$ & TCCA & 15 & $27,8 \%$ \\
\hline DIZDE & 12 & $22,2 \%$ & BCIPE & 6 & $11,1 \%$ & TMFL & 23 & $42,6 \%$ \\
\hline DINC & 3 & $5,6 \%$ & BJUPE & 1 & $1,9 \%$ & \multicolumn{3}{|c|}{ REEQUILIBRIO } \\
\hline \multicolumn{3}{|c|}{ POSICIÓN PIE DERECHO } & \multicolumn{3}{|c|}{ POSICIÓN CADERA } & $\mathrm{DA}$ & 11 & $20,4 \%$ \\
\hline PDDE & 11 & $20,4 \%$ & CISE & 16 & $29,6 \%$ & $\mathrm{DD}$ & 11 & $20,4 \%$ \\
\hline PDEX & 1 & $1,9 \%$ & CIFU & 2 & $3,7 \%$ & AMD & 1 & $1,9 \%$ \\
\hline PDID & 3 & $5,6 \%$ & CDME & 1 & $1,9 \%$ & ID & 5 & $9,3 \%$ \\
\hline \multicolumn{3}{|c|}{ POSICIÓN PIE IZQUIERDO } & CDFU & 1 & $1,9 \%$ & IA & 10 & \\
\hline PINI & 1 & $1,9 \%$ & \multicolumn{3}{|c|}{ ACCIÓN DE PIERNAS } & \multicolumn{3}{|c|}{ GLOBALIDAD } \\
\hline PID & 14 & $25,9 \%$ & APEX & 6 & $11,1 \%$ & LENT & 2 & $3,7 \%$ \\
\hline PIDM & 7 & $13 \%$ & APIF & 20 & $37 \%$ & & & \\
\hline
\end{tabular}

4

Tabla 2

Frecuencia y porcentaje de los errores técnicos en O Goshi 


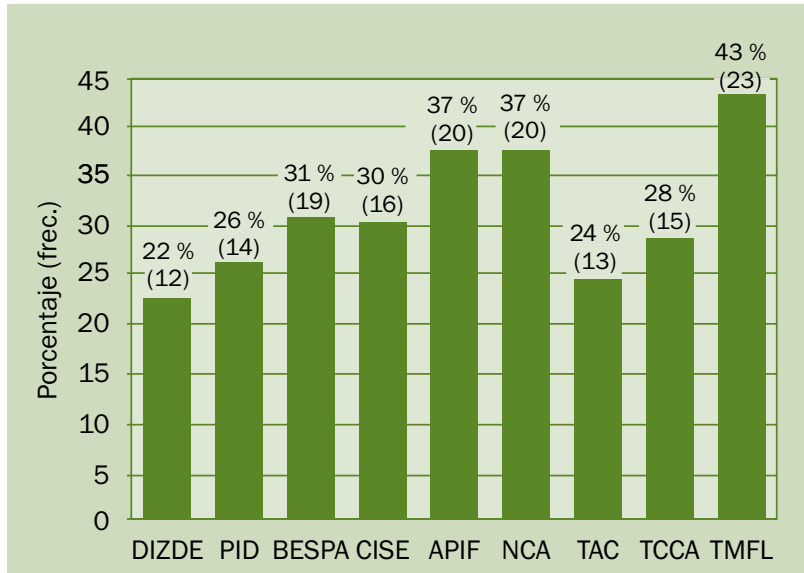

$\Delta$

Figura 5

Errores más frecuentes en la técnica O Goshi

pocos errores comunes y muchos errores poco frecuentes (fig. 5).

Los errores más comunes y constatados son: la falta de una acción de desequilibrio inicial sobre el adversario con ambos brazos -DIZDE-, el apoyo del brazo derecho a la región dorsal de la espalda de Uke tras el Tai Sabaki-BESPA-, el inadecuado posicionamiento de la cadera -CISE-, la ausencia de una óptima flexión de piernas -APIF-, y la falta de carga del cuerpo de Uke sobre la cadera de Tori-NCA-.
Finalmente, para comprobar la existencia de diferencias entre hombres y mujeres respecto de los errores observados en la ejecución de $O$ Goshi, se ha realizado un análisis de los datos mediante la prueba $U$ de Mann Whitney.

De este modo, solo se han obtenido diferencias estadísticamente significativas, entre ambos sexos $(p<0,05)$, en el error BESPA (Tori pasa su mano derecha por debajo de la axila izquierda del cuerpo de Uke, abrazando la región dorsal de la espalda de su adversario para proyectarle), siendo observado el mayor porcentaje de errores en los sujetos masculinos. Una posible explicación sería el hecho de que la mayor parte de los sujetos -hombres- que cometieron este error realizaron esta técnica con un Uke de un tamaño inferior al suyo, por lo que, a priori, y a consecuencia de su altura les resultó más complicado abrazar la región lumbar del oponente. Dicha circunstancia no sucedió así en las mujeres porque la mayor parte de estas utilizaron para la ejecución de $O$ Goshi un compañero de tamaño similar o superior.

\section{Detección de patrones temporales}

Con el fin de seguir profundizando en los errores más frecuentes de la técnica $O$ Goshi, se ha llevado a cabo un análisis secuencial de patrones temporales

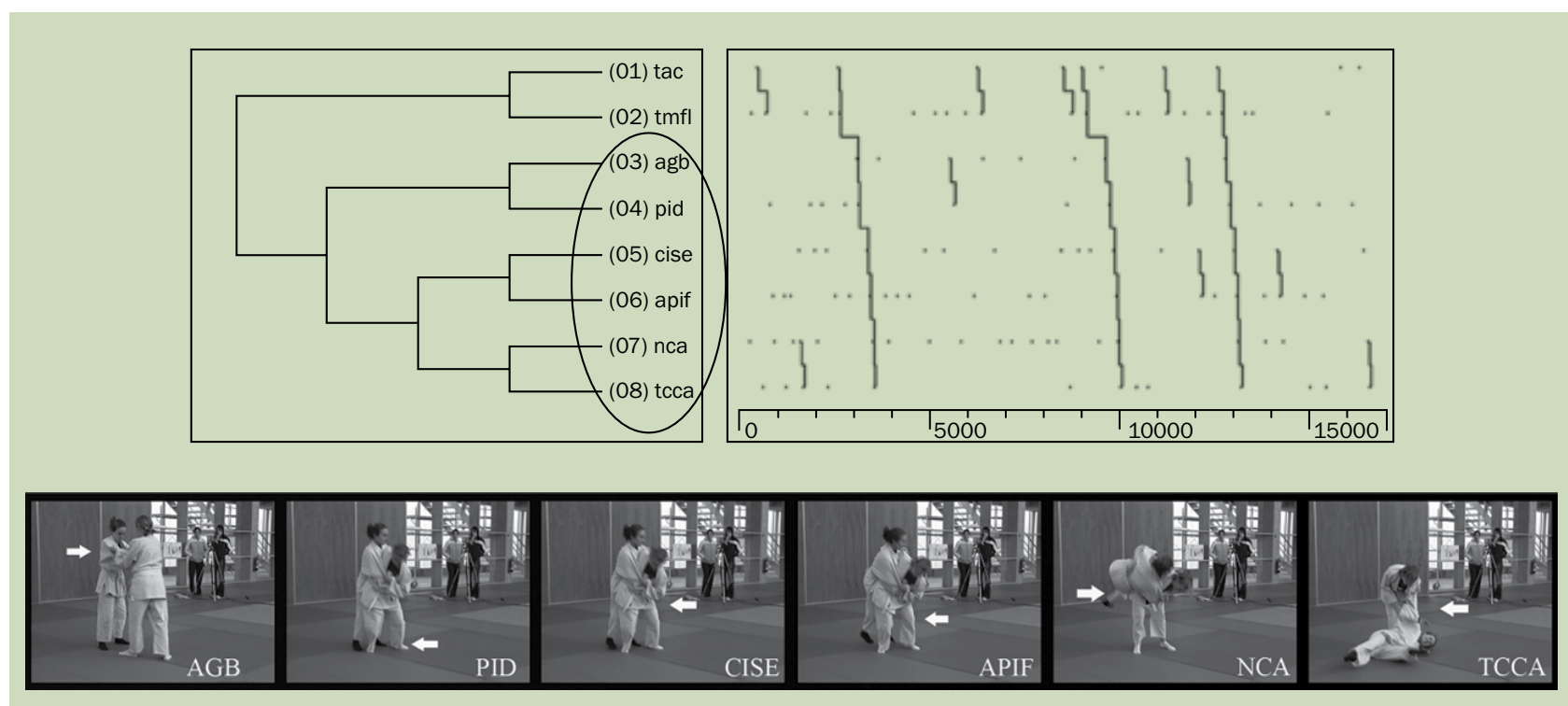

-

Figura 6

Primer T-Pattern de 0 Goshi 

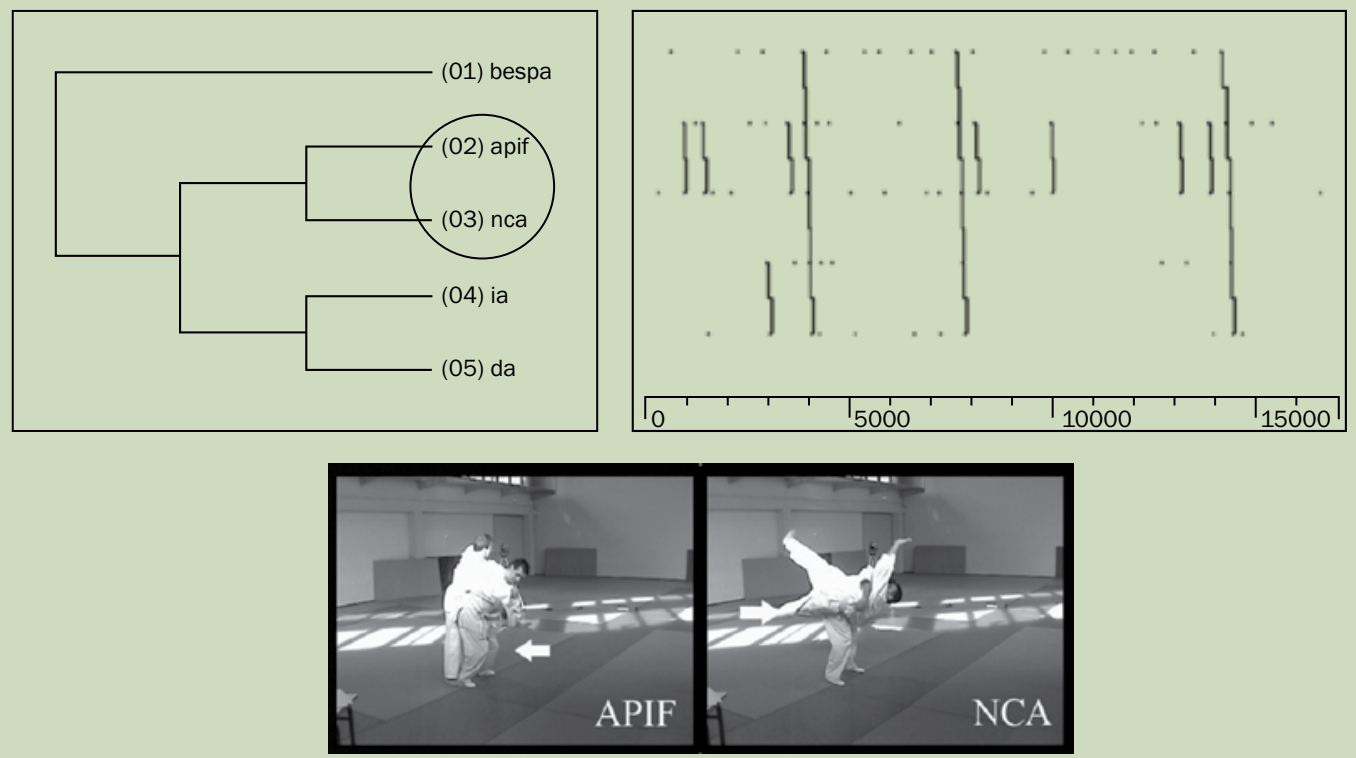

\section{Figura 7}

T-Pattern relacionado con los errores observados

de datos (Magnusson, 1996, 2000, 2006) mediante el programa Theme, que permite obtener y representar los dendogramas correspondientes a acciones compuestas de códigos concurrentes que suceden con unas distancias temporales entre sí (Anguera, 2005) y que permanecen relativamente invariables dentro del intervalo crítico previamente fijado.

En el análisis de los denominados T-Patterns (Patrones de conducta temporales), que representaremos mediante gráficos en forma de dendogramas, se manifiesta una serie de vinculaciones importantes relacionadas con la aparición de errores en cadena y que nos van a permitir profundizar en el conocimiento de la técnica $O$ Gosh $i$ (ver figs. 6 y 7 ).

En primer lugar, tal como muestra el T-Pattern (fig 6), se evidencia cómo la falta de una flexión óptima de piernas (APIF) provoca la ausencia de carga del cuerpo de Uke sobre la cadera de Tori (NCA). De la misma manera, el inadecuado agarre inicial (AGB), la falta de desequilibrio inicial con ambos brazos (DIZDE) -no presente en este dendograma pero sí en otros- o la separación de la parte posterior de la cresta iliaca izquierda del cuerpo de Tori sobre la parte anterior del hemicuerpo izquierdo de Uke (CISE) conducen a este mismo error. Además, se evidencia cómo una parte de los sujetos que ejecutan esta técnica, tras no cargar el cuerpo del oponente (NCA), no controlan tampoco la caída (TCCA).
Por otro lado, conviene señalar que en una gran parte de los casos estudiados, la flexión insuficiente de piernas (APIF) es precedida del error de apoyar el brazo derecho sobre la espalda del adversario (BESPA) al introducir dicho brazo por debajo de la axila del de este durante el movimiento de Tai Sabaki que Tori efectúa para situarse de espaldas a Uke. Asimismo, la falta de una flexión óptima de piernas (APIF) y errónea acción de carga (NCA), establece una vinculación importante con los errores que describen la acción de reequilibrio que el ejecutante realiza tras la ejecución de la técnica con el propósito de conservar su posición de equilibrio (IA-DA). En cualquier caso, lo más significativo de este dendograma, que muestra la figura 7, es comprobar cómo se confirma la importancia de la relación entre los dos errores que señalábamos anteriormente (APIF-NCA).

\section{Discusión}

Sin duda alguna, una de las secuencias principales acontecidas en esta investigación ha sido la relación existente entre la insuficiente flexión de piernas -o la ejecución de la proyección en completa extensión(Inogai \& Habersetzer, 2002; Daigo, 2005) y la falta de carga del cuerpo de Uke sobre la cadera del ejecutante (García, Del Valle, Díaz, \& Velázquez, 2006). 
Podemos interpretar que el hecho de no descender el centro de gravedad lo suficiente, mediante esa flexión óptima de piernas, conduce a no cargar óptimamente el cuerpo del rival al encontrarse las caderas a una misma altura. Esta situación obliga a realizar una gran fuerza con el brazo derecho para poder realizar correctamente dicha acción lo cual va en contra del principio de máxima eficacia en el uso de la fuerza Seiryoku Zenyo.

Por otro lado, aunque en la misma línea de la secuencia precedente, también parece lógico que algo similar ocurra cuando la cresta iliaca izquierda del cuerpo de Tori está separada -tras el Tai Sabaki- de la parte anterior del hemicuerpo izquierdo de Uke (Kobayashi \& Sharp, 1995; Ohlenkamp, 2006), pues la distancia entre los cuerpos no facilita, de ninguna manera, una acción ulterior de carga.

\section{Conclusiones}

Una vez finalizado el análisis estadístico descriptivo y el análisis secuencial de patrones temporales de la técnica $O G o s h i$ y tras un proceso de interpretación y discusión del mismo, las conclusiones de la presente investigación son las siguientes:

- Los errores que con más frecuencia comete este grupo de estudio en la ejecución de la técnica $O$ Goshi son la falta de un correcto desequilibrio inicial, la leve flexión de piernas que Tori realiza durante la ejecución de dicha proyección y la ausencia de carga del cuerpo de Uke sobre la cadera de Tori.

- La colocación errónea del brazo derecho de Tori sobre el cuerpo de Uke durante la fase de Tsukuri también es uno de los errores más habituales.

- Del mismo modo, es bastante usual que estos sujetos sitúen el pie izquierdo delante del mismo pie de Uke -en lugar de ubicarlo en el interior-.

- Asimismo, hemos constatado que muchos de los alumnos estudiados acompañan, con su brazo derecho, el cuerpo de Uke durante la fase final de la proyección hasta que este contacta con el suelo; y que, además, una vez ha sido proyectado Uke permanecen en flexión de tronco $70^{\circ}-90^{\circ}$ en lugar de recuperar la verticalidad.

- Los patrones de comportamiento de conductas secuenciales más importantes que hemos constatado son:
- La falta de flexión de piernas provoca la ausencia de carga del cuerpo de Uke sobre la propia cadera de Tori.

- La falta de desequilibrio inicial con ambos brazos o la separación de la parte posterior de la cresta iliaca izquierda del cuerpo de Tori sobre la parte anterior del hemicuerpo izquierdo de Uke conducen también a la ausencia de carga del cuerpo del adversario.

\section{Agradecimientos}

Este trabajo forma parte de las investigaciones:

- Avances tecnológicos y metodológicos en la automatización de estudios observacionales en deporte que ha sido subvencionado por la Dirección General de Investigación, Ministerio de Ciencia e Innovación (PSI2008-01179), durante el trienio 20082011 y del grupo de investigación consolidado de la Generalitat (2009-2013).

- Grup de recerca i innovació en dissenys (GRID). Tecnologia $i$ aplicació multimèdia $i$ digital als dissenys observacionals.

La investigación se ha desarrollado gracias a la ayuda concedida por la Universidad de Vigo para efectuar una estancia de investigación en el Laboratorio de Observación de la Motricidad del INEFC de Lleida (Ref.: 0022 122I 481.02 bolsas estadías).

\section{Referencias}

Anguera, M. T. (1999). Observación en deporte y conducta cinésicomotriz: aplicaciones. Barcelona: Edicions de la Universitat de Barcelona.

Anguera, M. T. (2005). Microanalysis of T-patterns. Analysis of symmetry/asymmetry in social interaction. En L. Anolli, S. Duncan, M. Magnusson, \& G. Riva (Eds.), The hidden structure of social interaction. From Genomics to Culture Patterns (pp. 51-70). Amsterdam: IOS Press.

Anguera, M. T., Blanco, A., \& Losada, J. L. (2001). Diseños Observacionales, cuestión clave en el proceso de la metodología observacional. Metodología de las Ciencias del Comportamiento, 3(2), 135-161.

Bakeman, R. \& Quera, V. (1992). SDIS: A sequential data interchange standard. Behavior Research Methods, Instruments \& Computers, 24(4), 554-559.

Bakeman, R. \& Quera, V. (2001). Using GSEQ with SPSS. Metodología de las Ciencias del Comportamiento, 3(2), 195-214. doi:10.3758/BF03203604

Blanco-Villaseñor, A. \& Anguera, M. T. (2000). Evaluación de la calidad en el registro del comportamiento: Aplicación a deportes de equipo. En E. Oñate, F. García-Sicilia, \& L. Ramallo (Eds.), Métodos Numéricos en Ciencias Sociales (pp. 30-48). Barcelo- 
na: Centro Internacional de Métodos Numéricos en Ingeniería.

Castañer, M., Torrents, C., Dinušová, M., \& Anguera, M. T. (2009). Instrumentos de observación ad hoc para el análisis de las acciones motrices en Danza Contemporánea, Expresión Corporal y Danza Contact-Improvisation. Apunts. Educación Física y Deportes (95), 14-23.

Castarlenas, J. L. \& Calmet, M. (1999). Aspectos conceptuales del judo: clasificación y enseñanza de sus contenidos. En M. Villamón (Dir.), Introducción al judo (pp. 263-290). Barcelona: Hispano Europea.

Castellano, J., Perea, A., Alday, L., \& Hernández Mendo, A. (2008). The Measuring and Observation Tool in Sports. Behavior Research Methods, 40(3), 898-905 doi:10.3758/BRM.40.3.898

Daigo, T. (2005). Kodokan Judo Throwing Techniques. Tokyo: Kodansha International.

Fernández, J., Camerino, O., Anguera, M. T., \& Jonsson, G. K. (2009). Identifying and analyzing the construction and effectiveness of offensive plays in basketball by using systematic observation. Behavior Research Methods, 41(3), 719-730.

García, J. M., Del Valle, S., Díaz, P., \& Velázquez, R. (2006). Judo. Juegos para la mejora del aprendizaje de las técnicas. Barcelona: Paidotribo.

García, J. M., Sterkowicz, S., \& Carratalá, V. (2003). Estudio sobre la dificultad de realizar técnicas de judo en la etapa infantil. Una nueva propuesta de Gokyo. En J. Campos (Coord.), III Congreso de la Asociación Española de Ciencias del Deporte. "Hacia la convergencia europea" [CD]. Valencia: Facultat de Ciències de l'Activitat Física i l'Esport, Universitat de València.

Gentile, A. M. (1972). A working model of skill acquisition with application to teaching. Quest, 17(1), 3-23.

Godoy, J. F. (1994). Biofeedback y deportes: potenciales líneas de actuación. Revista Motricidad, 1, 117-128.

Gutiérrez, A. \& Prieto, I. (2006). Errores en el modelo técnico deportivo en la iniciación al Judo: Morote Seoi Nague. Revista de educación física: Renovar la teoría y práctica (102), 29-34.

Gutiérrez, A. \& Prieto, I. (2007a). Las claves en el proceso de enseñanza-aprendizaje de la técnica de Judo desde la perspectiva del error: O Soto Gari vs. O Soto Guruma. Revista Motricidad, $18,93-110$.

Gutiérrez, A. \& Prieto, I. (2007b). Ippon Seoi Nague vs. Morote Seoi Nague: los 10 puntos básicos para su utilización en el proceso de enseñanza-aprendizaje desde la perspectiva del error. Revista de educación física: Renovar la teoría y práctica (105), 19-24.

Gutiérrez, A., Prieto, I., \& Cancela, J. M. (2009). Most frequent errors in judo uki goshi technique and the existing relations between them through T-patterns. Journal of Sports Science and Medicine 8(CSSI 3), 36-46.

Heinemann, K. (2003). Introducción a la metodología de la investigación empírica. Barcelona: Paidotribo.

Inogai, T. \& Habersetzer, R. (2002). Judo pratique. Du débutant à la ceinture noire. Paris: Amphora.

Jonsson, G. K., Anguera, M. T., Blanco-Villaseñor, A., Losada, J. L., Hernández-Mendo, A., Ardá, T., ... Castellano, J. (2006). Hidden patterns of play interaction in soccer using SOF-CODER. Behavior Research Methods, Instruments \& Computers, 38(3), 372-381.

Kobayashi, K. \& Sharp, H. E. (1995). The Sport of Judo. Japón: Charles E. Tuttle Company.

Lomas, C. (1996). El espectáculo del deseo: usos y formas de la persuasión publicitaria. Barcelona: Octaedro.

Magnusson, M. S. (1996). Hidden real-time patterns in intra- and interindividual behavior. European Journal of Psychological Assessment, 12(2), 112-12 . doi:10.1027/1015-5759.12.2.112

Magnusson, M. S. (2000). Discovering hidden time patterns in behavior: T-patterns and their detection. Behavior Research Methods, Instruments, \& Computers, 32(1), 93-110. doi:10.3758/BF03200792

Magnusson, M. S. (2006). Structure and communication in interaction. En G. Riva, M. T. Anguera, B. K. Wiederhold, \& F. Mantovani (Eds.), From Communication to Presence: Cognition, Emotions and Culture Towards the Ultimate Communicative Experience (pp. 127146). Amsterdam: IOS Press.

Ohlenkamp, N. (2006). Black Belt. Judo Skills and Techniques. London: New Holland Publishers (UK).

Oña, A., Martínez, M., Moreno, F., \& Ruiz, L. M. (1999). Control y aprendizaje motor. Madrid: Síntesis.

Ruiz, L. M. \& Sánchez, F. (1997). Rendimiento deportivo. Claves para la optimización de los aprendizajes. Madrid: Gymnos.

Schmidt, R. \& Lee, T. D. (2005). Motor control and learning. Champaign: Human Kinetics.

Taira, S., Herguedas, J., \& Román, F. I. (1992). Judo (I). Madrid: COE. 